\title{
Patrimonio y comunidad patrimonial: construcción de una identidad compartida en un entorno rural ${ }^{1}$
}

\author{
Xulia Marqués $\left({ }^{*}\right)$, Belén Castro-Fernández ${ }^{* *}$ y Ramón López-Facal $\left({ }^{* *}\right)$ \\ ${ }^{*}$ ) CPI Plurilingüe de Vedra- España - $\left(^{* *}\right)$ Universidad de Santiago de Compostela -España
}

\section{RESUMEN}

Se presenta una evaluación de un proyecto educativo basado en la identificación con el patrimonio cultural inmaterial de un centro situado en una comunidad rural. Se analizan los aprendizajes de los alumnos sobre el carnaval tradicional de la comarca del Ulla (A Coruña). Se valora en qué medida el proyecto tiene impacto en la sociedad y el proceso de identificación del alumnado con las tradiciones de su entorno. La experiencia se ha desarrollado fuera del horario lectivo, y forma parte de un proyecto más amplio iniciado en 2016. Se pretende promover la construcción de una identidad inclusiva en una comunidad rural a partir del patrimonio cultural local, basada en el diálogo intergeneracional. Para ello es fundamental la implicación de la comunidad con el centro educativo. Participaron 44 escolares de educación primaria y secundaria y 22 vecinos adultos. Para tomar datos se emplea una metodología mixta mediante cuestionarios, narrativa con representación gráfica y grupo de discusión. Los resultados muestran la importancia y eficacia de las comunidades de aprendizaje que trascienden el trabajo dentro de las aulas. La colaboración de familiares y vecinos permite compensar la falta de participación del profesorado.

Palabras clave: Evaluación de proyecto, Educación patrimonial, Educación basada en el lugar, Comunidad rural, Diálogo intergeneracional.

\section{Heritage and heritage community: building a shared identity in a rural context}

\section{ABSTRACT}

This paper presents an evaluation of an educational project based on the sense of identification with the immaterial cultural heritage of a school located in a rural community. The learning process of schoolchildren regarding the traditional carnival of the Ulla district (A Coruna) was analysed. The extent to which the project had an impact on the community and the process of the pupils' identification with the traditions of their surrounding area was assessed. The experiment was performed outside of school hours and forms part of a broader project which began in 2016. The aim was to promote the construction of an inclusive identity in a rural community via the use of aspects of the local cultural heritage and intergenerational dialogue. In order to achieve this, the involvement of the community with the school was essential. Forty-four pupils of primary and secondary ages and 22 local adults participated in the experiment. Questionnaires and registers of the participants' observations were used to gather data. The results show the importance and effectiveness of learning communities which transcend work carried out in the classroom. Teachers lack the incentive to become involved in such projects due to the fact that the workload and the extra effort required by such activities is not recognised or supported by the administration. However, the limited involvement of teachers was counteracted, to a large extent, by the collaboration of the local community.

Key words: Project evaluation, Heritage education, Place-based education, Rural community, Intergenerational dialogue.

ISSN: 0210-2773

DOI: https://doi.org/10.17811/rifie.49.1.2020.25-34

\footnotetext{
Agradecimiento al proyecto COMDEMO (EDU2015-65621-C3-1-R), del Plan Nacional de I+D+i del gobierno de España y fondos FEDER de la UE.
} 


\section{Introducción}

Cuando una comunidad participa de una celebración tradicional con arraigo, se estrechan los lazos entre sus miembros y se refuerza el sentimiento de identidad, de pertenencia al grupo. Compartir vivencias contribuye a mantener vivas pautas de expresión cultural (Marqués, 2013, 2014). Si a la experiencia compartida se añade una acción educativa que favorece la resignificación y transmisión de esa tradición, se establece una comunidad patrimonial (Convenio de Faro, 2005) unida por un pacto intergeneracional. Es deseable que el interés hacia el patrimonio local surja desde una perspectiva endógena, dentro de la comunidad y no por reconocimiento exterior. Los programas intergeneracionales entre la escuela y la comunidad facilitan el intercambio de recursos y aprendizajes para obtener beneficios individuales y sociales (Madariaga-Orbea et al., 2018; Hatton-Yeo y Ohsako, 2001). Contribuyen a la formación de una ciudadanía socialmente comprometida con su patrimonio (Martín y Cuenca, 2015).

$\mathrm{Al}$ orientar el (re)descubrimiento de los referentes culturales compartidos, de los que se apropia simbólicamente una comunidad para construir su identidad (Gómez-Redondo, 2012; López-Facal, 2019), se favorece su patrimonialización a través de una secuencia de procedimientos y actitudes (Fontal, 2003). La dimensión comunitaria de este proceso requiere de un proyecto educativo que englobe aprendizaje significativo y contextualizado, trabajo actitudinal, participación y experimentación colectiva (Fontal y Gómez-Redondo, 2015). Esta acción se puede iniciar a edades tempranas, en las que se inicia la comprensión espacial y temporal. Es más fácil despertar la curiosidad infantil y adolescente hacia el patrimonio a través de las emociones, que empleando discursos sobre la relevancia del mismo. La aprehensión emocional del patrimonio permite establecer vínculos entre la sociedad y su identidad, tradiciones y paisajes (Cuenca-López y Estepa-Giménez, 2017).

La experiencia vivencial basada en el lugar y asociada a la comunidad, con significados colectivos y enlazado con la cultura material (Carrión, 2015) permite fortalecer los vínculos históricos relacionados con las manifestaciones culturales inmateriales. La cooperación comunidad-escuela facilita que los cambios y continuidades de la tradición local sean fruto de una decisión compartida y construida de manera consciente e informada.

Este estudio evalúa la eficacia de un proyecto de educación patrimonial de este tipo. Pretende dar respuesta a la siguiente pregunta de investigación: ¿en qué medida es posible realizar un proyecto de centro sobre educación patrimonial que implique a la comunidad local, a pesar de las limitaciones derivadas de las rutinas escolares? Se presentan resultados de la investigación sobre el proyecto de innovación educativa desarrollado en el Centro Público Integrado (CPI) de Vedra durante el curso 2018-2019, en torno al carnaval tradicional de la comarca del Ulla (A Coruña) titulado O Entroido rural tradicional na Ulla. Se pretenden generar, desde la escuela, procesos de conocimiento, puesta en valor y sensibilización hacia esta fiesta tradicional, presente en tres de las doce parroquias del municipio. El objetivo es que la comunidad escolar desarrolle una identidad territorial compartida, mediante la comunicación intergeneracional. La experiencia se desarrolla durante 5 sesiones, con participación de 22 vecinos adultos y 22 estudiantes de primaria y secundaria que participan voluntariamente fuera del horario lectivo.

Los objetivos específicos son los siguientes:
1. Identificar la motivación de la participación voluntaria, tanto del alumnado como de los agentes externos en actividades desarrolladas fuera del horario escolar.

2. Indagar sobre el papel que ambos colectivos atribuyen a la tradición para la conservación del patrimonio local.

3. Caracterizar el significado que los participantes otorgan a una identidad compartida.

\section{Escuela, territorio y comunidad: un proceso de patrimonia- lización}

Vedra es un municipio rural, de poco más de 5.000 habitantes, limítrofe con el de Compostela. Debido a esta proximidad está convirtiéndose también una zona residencial y con creciente peso de actividades de servicios e industriales que coexisten con las actividades agrarias. Cuenta con dos centros educativos, de carácter público, una escuela infantil (de 0 a 3 años) y un Centro Público Integrado (CPI) en la que se imparte educación infantil, primaria y secundaria obligatoria (de 3 a 16 años). En el CPI se desarrolla desde 2016 un proyecto integrado, Vedra Medra ${ }^{1}$, coordinado por la primera autora. Participan escolares de las tres etapas educativas, con actividades diferenciadas, pero con el objetivo común de favorecer su identificación con el territorio y la sociedad en la que viven. En estos años han desarrollado diferentes actividades basadas en el territorio: sobre la toponimia; el patrimonio material; oficios y tradiciones locales, y se incorporan cada año las memorias de personas que han sido testigos de las mudanzas una sociedad en la que van cambiado las actividades vinculadas al sector primario (agricultura, pesca) en un marco creciente de globalización económica. Desde el CPI se promueve el conocimiento sistemático de su territorio con visitas a cada una de sus parroquias, y de las tradiciones y memorias que se mantienen como señas de identidad en el imaginario colectivo. Para ello cuentan con la colaboración de personas, familiares o no de los estudiantes, asociaciones culturales y cívicas; y apoyo municipal.

El proyecto del CPI de Vedra se basa en un diseño de educación basado en el lugar (PBE, en sus siglas en inglés: Place Based Education). El término PBE, acuñado en los años 90 del pasado siglo, entronca con una tradición anterior que conecta con los planteamientos pioneros de Pestalozzi, Ferrer i Guardia o Dewey. Posteriormente, se ha ido desarrollado sobre todo en propuestas de educación ambiental (Elfer, 2011). Sobel (2014) ha sido probablemente el principal impulsor de la PBE "convencido de que el cambio mejorará tanto el aprendizaje de los estudiantes como el bienestar comunitario de las personas y el medio ambiente en el contexto local" (Sánchez Contreras y Murga-Menoyo, 2019, 159). Adoptar el enfoque de PBE implica llevar a cabo una intervención educativa que parta del contexto social y espacial de los escolares.

Identificarse con la comunidad en la que cada uno vive implica compartir emociones, que influyen en la manera en la que las personas interactúan entre ellas y con su entorno (Barraza y Castaño, 2012). Domínguez y López $(2017,50)$ afirman que:

Promover una conexión con el entorno facilita al alumnado sentirse activo y creativo en la comunidad, estableciendo vínculos con un paisaje entendido como patrimonio en su totalidad o mediante los elementos que lo articulan: se facilitará así la construcción de conciencia ciudadana o identidades sociales (Busquets, 2010; Domínguez y López, 2014, 2015a). La educación

http://www.edu.xunta.gal/centros/cpidevedra/taxonomy/term/124. 
a través del valor del paisaje puede contribuir a desvirtuar la extendida asociación consumo-felicidad.

La UNESCO (2012) ha reiterado la importancia de utilizar en la educación los recursos que proporcionan contextos próximos, tanto sociales como espaciales. Desde la Educación Ambiental se ha tendido a poner el foco sobre todo sobre los recursos naturales presentes en los paisajes. Sin embargo, las perspectivas de la PBE van más allá y prestan atención a todo tipo de elementos (Peterson, 2018). Como señalan Sánchez Contreras y Murga-Menoyo (2019, p.159):

El énfasis que cabe destacar en el PBE es su visión sistémica; la consideración del sistema-vida, con sus dimensiones ecosociales íntimamente interrelacionadas y su llamada de atención hacia la doble condición del humano, como ser ecodependiente $\mathrm{y}$, a la vez, interdependiente de los restantes seres vivos, en especial de sus congéneres.

Sánchez Contreras y Murga-Menoyo asumen las principales características de la educación basada en el lugar (PBE) que han sido caracterizadas por Woodhouse y Knapp $\left(2000,4^{2}\right)$ :

Emerge de las características concretas de un lugar. Incluye contenidos específicos de geografía, ecología, sociología, política y otras dinámicas de ese lugar. Esta característica fundamental es esencial en el concepto de PBE.

Por su naturaleza es multidisciplinar.

Es intrínsecamente experiencial. En muchos programas se incluye una acción participativa o un componente de aprendizaje de servicio; de hecho, algunos promotores consideran que la actividad debe contribuir a lograr la sostenibilidad ecológica y cultural.

Refleja una filosofía educativa cooperativa que va más allá de aprender "a ganar". Puede ocuparse de estudiar la economía local al contemplar la sostenibilidad económica; sin embargo, todos los proyectos están diseñados para objetivos más amplios.

Conecta el lugar con uno mismo y con su comunidad. Estas conexiones son dominantes en tanto que los proyectos educativos basados en el lugar asumen una perspectiva ecológica. Interactúan con los recursos de la comunidad por lo que incluyen dimensiones multigeneracionales y multiculturales.

El proyecto educativo Vedra Medra contribuye a la visibilidad y protección de los bienes patrimoniales y ha sido reconocida con diversos premios a la innovación educativa por diferentes instituciones. Uno de los materiales derivados de la actividad sobre el Entroido da Ulla, ha sido galardonado en el I Concurso de Podcasts, convocado por la Xunta de Galicia en colaboración con la RTVG. Focalizar el proyecto en el entroido (carnaval) se justifica por su carácter interactivo, por su enorme arraigo en la comarca, pero también por contribuir a su continuidad, ya que actualmente sólo permanece vivo en tres de las doce parroquias del municipio.

\section{Método}

La hipótesis inicial es que la realización de un proyecto de centro sobre patrimonio local que implique a la comunidad facilita la construcción de una identidad compartida desde una perspectiva intergeneracional. Para contrastarla, se aplicó una metodología de investigación mixta.

\subsection{Instrumentos}

Para la recogida de información se diseñaron cuatro instrumentos. Un cuestionario y un ejercicio de narrativa con repre-

Traducción del original, nuestra. sentación gráfica entre el alumnado, desde $3^{\circ}$ de Primaria a $4^{\circ}$ de Secundaria. A los adultos que participaron en el proyecto, se les entregó otro cuestionario y, con algunos de ellos se organizó un grupo de discusión:

\section{Cuestionario al alumnado.}

2. Narrativa con representación gráfica para los escolares.

3. Cuestionario a los adultos que participaron en el proyecto.

4. Grupo de discusión con adultos que participaron en el proyecto.

Los instrumentos han sido validados en sus aspectos de forma, estructura y contenido por cuatro docentes de las universidades de Murcia, País Vasco y Santiago de Compostela, expertos en educación patrimonial e iniciativas didácticas en museos; didáctica de la historia y formación docente con especial interés en patrimonio e historia local; interrelación escuela-comunidad y procesos de patrimonialización desde la antropología social y cultural; y métodos de investigación desde la psicología social. El proceso de validación permitió un diseño más asequible para los diferentes participantes. El instrumento uno ha incorporado más modificaciones, para simplificar las respuestas de los escolares y poder categorizar, posteriormente, los resultados. Se reformularon siete preguntas de elección múltiple, y en otras tres se incluyó una tabla para ordenar informaciones. Cinco preguntas mantuvieron el diseño inicial y se añadió una nueva para profundizar en la concepción patrimonial de los jóvenes. El enunciado del instrumento 2 se alteró parcialmente para facilitar las respuestas, por la dificultad de preguntar al alumnado por posibles escenarios futuros. El instrumento 3 apenas se modificó, únicamente se incluyó una tabla para ordenar alguna respuesta. Al igual que en el instrumento 1, se añadió una pregunta nueva y similar a la planteada en aquel, para poder comparar ambos conjuntos. Teniendo en consideración la flexibilidad del instrumento 4 , únicamente se reformuló una pregunta formulada de manera directa.

\subsection{Caracterización de la muestra}

En los instrumentos 1 y 2 se ha realizado un muestreo probabilístico, de tipo aleatorio por conglomerados (Bisquerra, 1989; Buendía, Colás y Hernández, 1997). En los instrumentos 3 y 4 se trabaja con una muestra intencional (Deslauriers, 2004).

Instrumento 1: Cuestionario al alumnado. Se pasó a 258 estudiantes desde $3^{\circ}$ curso de Primaria a $4^{\circ}$ de Secundaria que suponen el $70 \%$ del total registrado en el municipio. Es una muestra equilibrada, con ligera mayoría de varones (56\%); mantiene una proporción casi exacta a la distribución del alumnado total del ayuntamiento. Se distribuyó prácticamente igual entre los cuatro cursos de Primaria (48\%) y ESO (52\%), con una distribución casi equivalente entre grupos de edades, de 9 a 11 años (36\%), entre 12 y 14 años (40\%), siendo el grupo de más de 15 años (24\%) el menor. Un $25 \%$ de los escolares residen en ayuntamientos limítrofes.

Instrumento 2: Ejercicio de narrativa con representación gráfica. La información obtenida con este instrumento es cualitativa, se trata de una muestra no igual y más equilibrada en términos de género, pero muy semejante a la del instrumento 1, con 219 informantes: $47 \%$ en cursos de Primaria y 53\% de ESO. Por edades: 
31\% de 9 a 11 años; 40\% entre 12 y 14 años; y 29\%) de 15 y más años. Por lugar de residencia, tres cuartas partes corresponde a Vedra $(74 \%)$ y el resto, a otros municipios limítrofes.

Instrumento 3: Cuestionario a adultos que han participado en el proyecto. Han respondido 17 personas. Promedio de edad, 50 años. 12 mujeres. Casi un tercio no residen en Vedra.

Instrumento 4: Grupo de discusión con adultos que participan en el proyecto. Se ha limitado el número para facilitar la intervención de todos ellos, aunque suficiente para incorporar deferentes perfiles: 6 adultos de unos 50 años de edad, 5 de ellos, mujeres; cuatro no residentes en Vedra.

\subsection{Procedimiento}

\section{$1^{a}$ Fase. Desarrollo del taller fuera de horario lectivo}

Se ha desarrollado en cinco sesiones de 90 minutos de duración entre el 30 de octubre y el 15 de enero. Ha sido impartido por personas nacidas o residentes en Vedra que participan (o han participado) en el entroido de modo activo, en calidad de generales, correos, miembros de coros (de bonito; de vello) o confeccionando indumentaria y complementos para figurantes o para los caballos. También han intervenido personas mayores aportando testimonios en forma de relatos, trajes y fotografías.

Las sesiones se organizaron en torno a cinco centros de interés. La primera sobre la indumentaria de distintos personajes que concurren en el entroido tradicional (correos, generales, coros, abanderado, escuadra de gastadores), su confección, precio y evolución de los diseños. La segunda sobre los complementos para personas (banda, fajin, medallas, charletera, boina, tricornio, gorreta) y caballos (silla, manta, pechopetral, baticola, espejos). La tercera sobre la evolución de la fiesta, las antiguas rivalidades entre municipios vecinos y su reflejo en los atranques (desafíos en verso). La cuarta sobre la participación de la mujer en los últimos cincuenta años. La quinta, dedicada a revisar las coplas (atranques y composiciones para los coros) que se representaron en el CPI con participación de todo el alumnado de ESO que reprodujo un entroido tradicional. En esta última sesión se ha incluido un espectáculo de títeres, Eu tamén son xenerala, de la compañía Títeres Cachirulo.

\section{$2^{a}$ fase. Recogida de datos sobre el impacto socioeducativo}

Distribución del cuestionario (instrumento 1) al grupo experimental (participantes en el proyecto) y al de control, para poder comparar sus percepciones y aprendizajes. Se pretende identificar: motivaciones de la participación en los talleres organizados fuera del horario escolar; contribución de los mismos para el conocimiento y comprensión de la fiesta; y, la consideración del entroido como un patrimonio frágil, con riesgo de desaparecer. También se ha recogido información sobre el papel dicotómico mujer/hombre y autóctono/forastero para analizar valores inclusivos y de educación para la igualdad.

Simultáneamente se ha distribuido al grupo experimental y al de control un ejercicio de narrativa con representación gráfica (instrumento 2) para poder comparar cómo caracterizan la fiesta y su sensibilización patrimonial. Se les pidió que expresasen de forma libre sus preferencias sobre el futuro del entroido da Ulla, indicando elementos que consideren necesario mantener y los que no importa que cambien en el futuro. Podían acompañar la narrativa con un dibujo. Con ello se han obtenido datos sobre el papel que atribuyen a la tradición para la conservación del patrimonio local.
Se ha distribuido otro cuestionario entre los adultos que participaron de manera activa (instrumento 3 para identificar los motivos de su participación voluntaria, indagar en el papel que atribuían al entroido como elemento de cohesión social, caracterizar el significado que asignan a los componentes de la fiesta, así como revelar su percepción sobre el papel de la mujer y las personas no oriundas de la comarca en esta celebración.

Por último, se organizó un grupo de discusión con algunos adultos que han participado (instrumento 4), para cruzar testimonios sobre su percepción holística del entroido. Se limitó a una hora de duración para evitar temas irrelevantes y asegurar su atención. Se plantearon preguntas abiertas diseñadas previamente para contrastar la comprensión de la realidad que se estaba estudiando (McMillan y Schumacher, 2005). Las preguntas versaron sobre sentimiento, experiencia, opinión e información. Se trataba de penetrar en el contexto local y el ámbito personal de las personas que participaron en el proyecto intergeneracional sobre el entroido.

\section{$3^{a}$ Fase. Evaluación}

Se ha aplicado un enfoque mixto y complementario, cuantitativo y cualitativo. Para el análisis de los resultados obtenidos con los instrumentos 1 y 3 se utilizaron variables sociodemográficas para detectar posibles diferencias en función del género, edad o municipio de residencia.

La primera pregunta del cuestionario 1 busca comprobar el impacto de la participación activa: Hace unas semanas se celebraron en el colegio varias actividades relacionadas con el Entroido da Ulla ¿Has participado activamente en ellas? a) sí; b) sólo como espectador; c) no. En el instrumento 3 se utilizó la segunda cuestión: ¿Por qué has decidido participar en la actividad del colegio sobre el entroido? Elige la que consideres más importante: a) Porque fue una petición de alguna profesora con la que tengo buena relación; b) Porque tengo algún hijo o hija (u otro familiar) en el colegio; $c$ ) Porque me parece importante que las niñas y los niños conozcan las tradiciones de Vedra $y$ las conserven en el futuro.

En el instrumento 2, más complejo de analizar, se combinaron técnicas cuantitativas y cualitativas para garantizar la menor pérdida posible de información. Se estableció una primera codificación de los datos, o destilado conceptual, para detectar posibles tendencias según género, edad o parroquia de residencia. Se buscaba que los informantes señalaran aspectos del entroido que les gustaría mantener o cambiar (Imagina como te gustaría que fuera el entroido do Ulla dentro de muchos, muchos años. Señala que elementos quieres que se mantengan y cuales no te importa que cambien. Puedes acompañar tu relato con un dibujo) por lo que estos conceptos se convirtieron en vectores del análisis. De esta forma se pudieron codificar las narrativas según el principio rector de su discurso, tanto si aparecía de forma expresa como latente. Se clasificaron así las narrativas entre las que preferían que la fiesta continuara como está, las que mantendrían algunos elementos y cambiarían otros, y aquellas que estructuraron su discurso a partir de los cambios que harían. Establecidos estos códigos, se realizó el análisis cuantitativo de las narrativas, elaborando cruces con las principales variables sociodemográficas, de los que solo se encontraron diferencias significativas en el cruce por grupos de edad $\left[\chi^{2}=53,666(\right.$ Sig. $=.000)$ cruzando "preferencias sobre el futuro" y edad]. A continuación, se analizaron nuevamente las respuestas para identificar los elementos que mantendrían o cambiarían, con un doble objetivo: conocer la presencia y el peso en el discurso de elementos característicos del entroido da Ulla y caracterizarlos, posteriormente, a partir de los aspectos de la celebración con los que se relacionan. 
La representación gráfica de la fiesta completó la otra dimensión de análisis del instrumento 2. Debido a la heterogeneidad de los contenidos representados en los dibujos, se decidió clasificarlos por su relación o no con el entroido o con su celebración. Finalmente, se analizaron los aspectos concretos que reflejaban.

La sesión grupal del instrumento 4 se registró mediante grabación de video y audio. El tratamiento datos obtenidos se codificó tomando como referencia las preguntas previstas para su discusión y otras emergentes durante su curso. Se clasificaron los testimonios cruzados. El proceso de destilado permitió obtener finalmente la sistematización temática de los resultados.

\section{Resultados}

Los participantes han valorado el taller satisfactoriamente. La mayoría reconoce el valor patrimonial de esta tradición y su capacidad para reforzar los lazos comunitarios. Los resultados corroboran la importancia de crear comunidades de aprendizaje llevando la educación patrimonial fuera del aula. Coinciden en identificar amenazas y dificultades para la conservación del entroido, como la complejidad que suponen las diferentes particularidades locales, aunque reconocen su valor, pero pueden fragmentar la percepción de la festividad como un todo; otra dificultad es la alta exigencia organizativa y creativa que reclama una elevada participación comunitaria. Se han podido caracterizar también intereses y conocimientos en cada etapa educativa: el grupo de 9 a 11 años muestra interés y voluntad de aprendizaje sobre el entroido y, en términos globales, sabe reconocer y situar los elementos patrimoniales característicos; el de 12 a 14 expresa mayor implicación, y también sabe reconocer y valorar sus elementos patrimoniales; y el de más de 15 años refleja algún menor interés e implicación, aunque es el que más conocimiento muestra sobre el entroido, y el que más lo valora en términos de patrimonio y conservación de la tradición.

Objetivo 1. Identificar la motivación de la participación voluntaria en las actividades desarrolladas fuera del horario escolar.

\section{Motivación, y experiencias previas}

Casi un 33\% de los escolares señala que la principal razón para asistir a la actividad es la participación de otros compañeros/as, seguida de la curiosidad por saber más del entroido (23\%) y haber disfrutado previamente de otras actividades organizadas desde el proyecto Vedra Medra (17\%). Sólo se aprecian dife- rencias significativas por grupos de edad $\left[\chi^{2}=34,93(\right.$ Sig. $=.000)$ para el cruce entre p1.1 y edad]: a medida que ésta aumenta se refleja más motivadora la asistencia de sus amistades.

Para la mayor parte de los adultos es su primera participación en actividades organizadas por CPI. Solo el 29\% cuenta con algún tipo de experiencia previa en otros talleres del programa Vedra Medra. Su principal motivación es la transmisión intergeneracional, que los niños y las niñas mantengan viva la tradición para la conservación del patrimonio (Gráfico 1). También destaca el hecho de tener hijos/as u otros familiares en el centro escolar, seguida de la petición de algún docente para asistir a los talleres.

\section{Formas en las que la tradición del entroido está viva en las familias}

Tres de los adultos participantes en el grupo de discusión cuentan con una larga tradición familiar en torno al entroido, como proveedores de trajes o artífices de los atranques (coplas populares de esta fiesta). Sus testimonios permiten indagar en las formas en que esta tradición se mantiene viva, de ahí su interés por participar en los talleres escolares. Consideran también muy importante que las familias transmitan esta tradición.

Objetivo 2. Indagar sobre el papel que atribuyen a la tradición para la conservación del patrimonio local

\footnotetext{
Razones para que la tradición perviva solamente en algunas parroquias
}

La mayoría del alumnado, con excepción del grupo de 9 a 11 años que no tiene un conocimiento claramente localizado de las parroquias de Vedra, identifica en las que se celebra el Entroido tradicional da Ulla de manera intensa: San Xián de Sales, Santo André de Trobe y San Pedro de Vilanova. Contrasta con la percepción de quienes no participaron, que señalan otras en las que no está tan presente esta tradición. El grupo de control considera mayoritariamente que la razón principal por la que no se festeja en algunas parroquias es la falta de asociaciones destinadas a su organización, mientras que el grupo experimental considera en mayor medida que se debe, simplemente, a que dejaron de celebrarse, aunque sin restar importancia al papel de las asociaciones.

Más de la mitad de los adultos coincide con el alumnado en que el entroido dejó de celebrarse en algunas parroquias porque se perdió la tradición. Para casi la cuarta parte el principal mo-

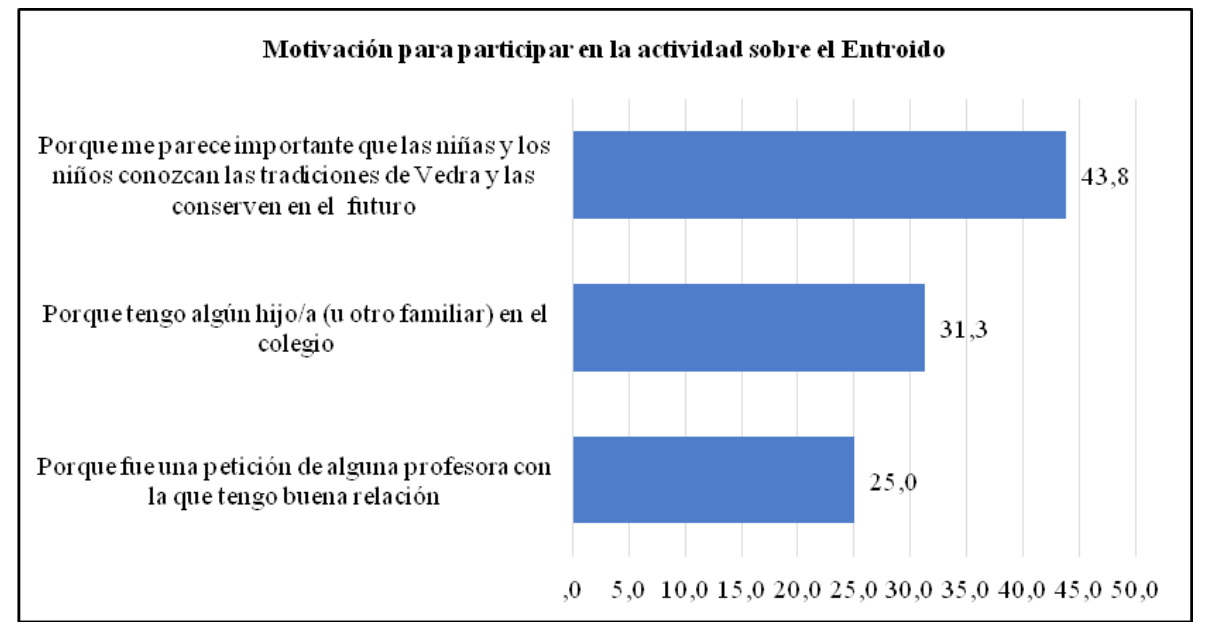

Grafíco 1. Motivación de los adultos para participar en la actividad sobre el Entroido do Ulla 
tivo se encuentra también en la inexistencia de asociaciones vecinales, responsables de organizar las fiestas. Algunas personas indican que un factor importante para su abandono en algunos lugares ha sido falta de reconocimiento del esfuerzo que conlleva su organización, con una gran diversidad de trajes, caballos, participación de jóvenes y mayores, ensayos de canciones, etc. En el grupo de discusión se ha coincidido en ello.

\section{Importancia de divulgar y dar a conocer el Entroido do Ulla}

El alumnado considera mayoritariamente que este tipo de actividades les permite conocer mejor su municipio $(63 \%)$, porcentaje que es mayor en el grupo experimental (70,8\%) que en el de control $(57,1 \%)$. En cuanto a su contribución al conocimiento general sobre el Entroido do Ulla, los resultados son similares: la mayoría considera que gracias a estas actividades puede aprenderse más sobre esta tradición (Gráfico 2).

¿Los escolares que partip aron en el taller sobre el Entroido do Ulla aprendieron más que aquellos que no? $(\%)$

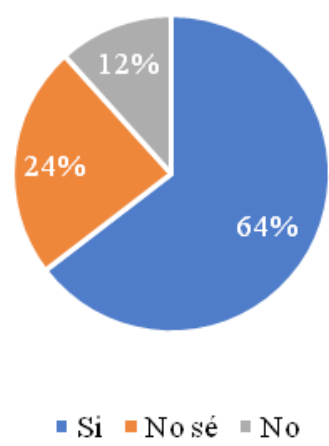

Gráfico 2. Aprendizaje del grupo experimental sobre el Entroido do Ulla

Los resultados no muestran diferencias por género y procedencia, pero sí por grupos de edad (Gráfico 3). En el de los más pequeños existe mayor confianza en el aprendizaje que pueden adquirir con estas actividades, un resultado consistente con que este grupo de edad señale como la razón más importante para participar en el taller es aprender más sobre esta fiesta. Las diferencias por edad pueden explicarse por una mayor curiosidad entre los más jóvenes, y el superior conocimiento sobre el entroido entre los escolares mayores.

Tres cuartas partes del alumnado señalan que les gustaría que se repitieran más veces este tipo de actividades. Casi el 69\% del grupo experimental repetiría en una nueva ocasión, mientras que solo el 37\% del grupo de control cambiaría de idea y participaría en nuevas actividades. Dentro del grupo experimental, quienes consideran que aprendieron son mucho más favorables a repetir $(74 \%)$, que los que dicen que no aprendieron nada nuevo, que solo repetirían 3 de cada 10. La valoración positiva del propio aprendizaje incide favorablemente en el deseo de repetir este tipo de actividades. La edad también presenta una correlación estadísticamente sólida, ya que el grupo del 12 a 14 años tiende a participar más y a estar más implicado y, en este caso, más interesado en las actividades relacionadas con el entroido. $\mathrm{Al}$ responder sobre sus principales referentes en el aprendizaje sobre el entroido, hasta un 55\% considera a sus profesoras como las personas de las que más aprenden, seguido, con bastante diferencia, por lo aprendido en la casa (Gráfico 4).

Los adultos consideran que una de las principales vías para trabajar con el alumnado las tradiciones y las costumbres de su parroquia, es lograr que el vecindario se implique en las actividades que organizadas desde el colegio (Gráfico 5). Manifiestan que son las personas que viven allí las que más pueden aportar sobre la historia, costumbres y tradiciones y deben ser parte activa en la educación si quieren que perviva su patrimonio cultural.

\section{Elementos fundamentales para mantener viva la tradición del Entroido}

El instrumento 2 permitió comprobar que prácticamente la mitad del alumnado prefiere que el entroido se mantenga sin cambios (49\%); más de una tercera parte modificaría algunos aspectos $(36 \%)$, y sólo un $12 \%$ lo cambiaría sensiblemente, proponiendo actividades sin vinculación con esta fiesta como transformarlo en una guerra de láseres o en una batalla entre científicos destacados. Una vez agrupados los discursos a partir de las categorías de respuesta, se buscaron correlaciones con las principales variables sociodemográficas, encontrando solo diferencias significativas en el cruce por grupos de edad $[\chi 2=53,666$ $($ Sig. $=.000)$ y sus "preferencias sobre o futuro" (Gráfico 6).

El grupo de 15 y más años prefiere conservar el entroido como es actualmente, probablemente por una mayor conciencia de su significado. El de 12 a 14 indica mayoritariamente que haría algún

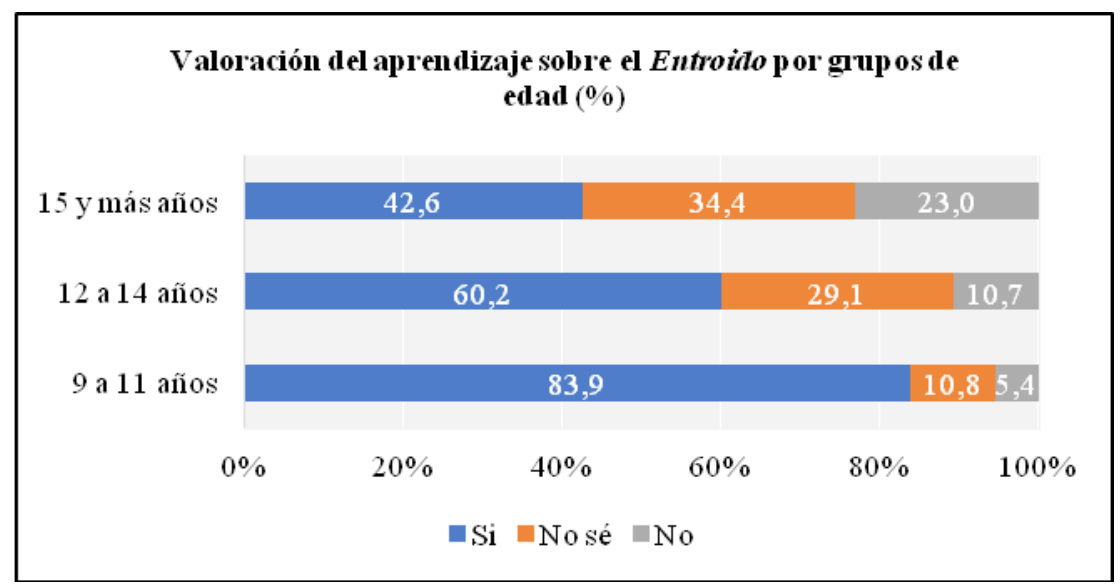

Gráfico 3. Valoración del aprendizaje adquirido por los escolares sobre el Entroido por grupos de edad 


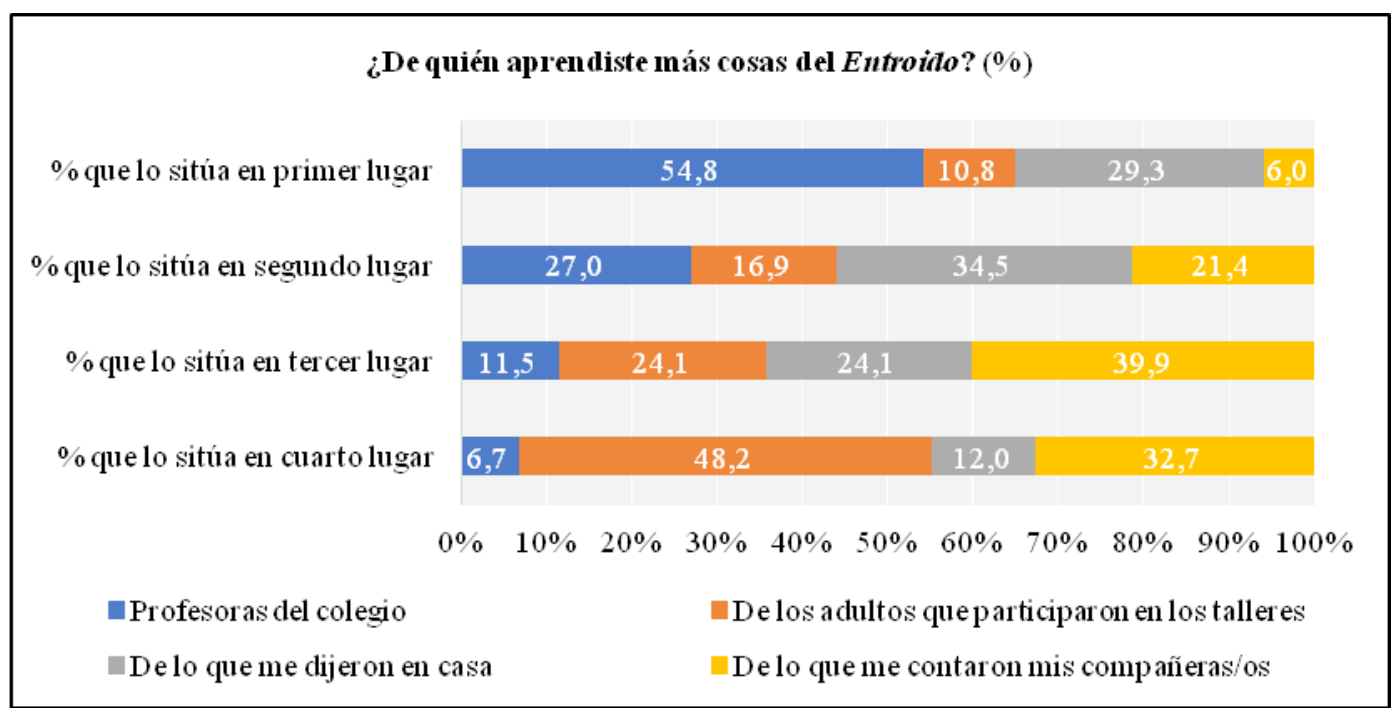

Gráfico 4. Principales referentes en el aprendizaje de los escolares sobre el Entroido

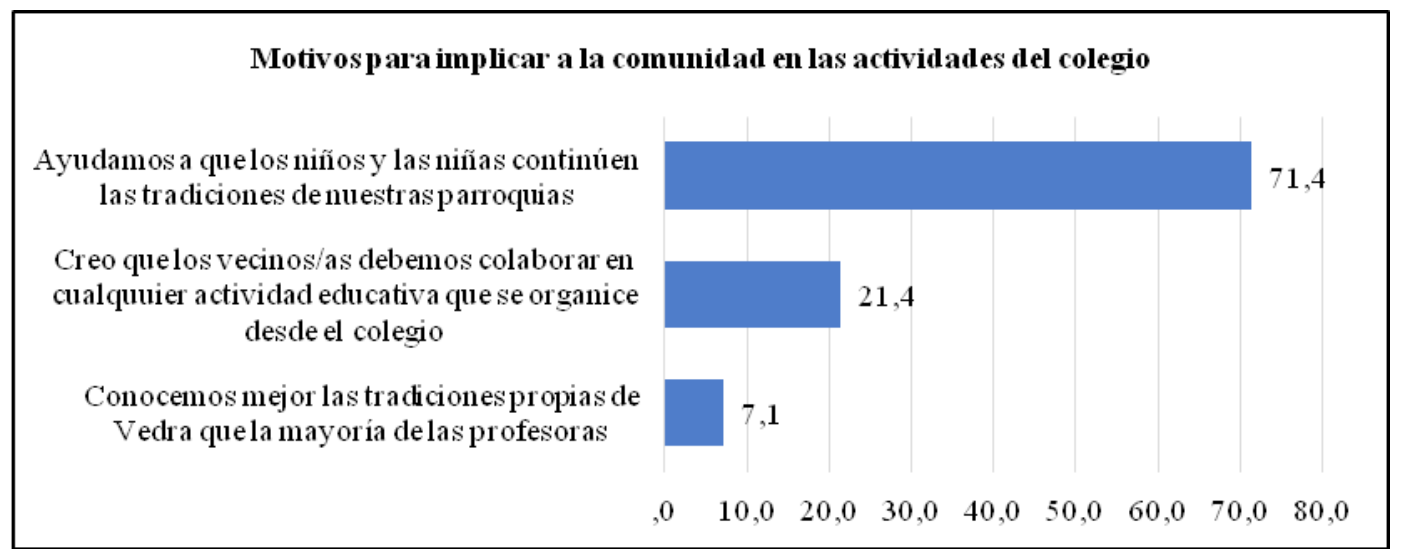

Gráfico 5. Principales vías que los adultos señalan para trabajar las tradiciones con los escolares

tipo de cambio, medio o profundo. Una cuarta parte de los que contestan que la mantendrían igual o parcialmente algunos de sus aspectos, se divide, casi a partes iguales, entre quienes formulan razones vinculadas a una preferencia subjetiva ("me gusta así" es la respuesta del $47 \%$ ) y quienes lo justifican para preservar la tradición (53\%: "es una tradición que debe mantenerse").

Buena parte de los elementos que el alumnado desea mantener están directamente vinculados a personajes o aspectos característicos. La mayoría sabe caracterizar este patrimonio cultural y valorar también sus elementos específicos. Los aspectos que consideran que deberían cambiar tienden a estar más relacionados con la forma en la que se desarrolla la celebración. Por ejemplo, entre otros, indican aspectos vinculados a la participación de la gente, de las mujeres o del resto de las parroquias, al tipo de música o comida; todo ello más relacionado con la forma que con el fondo del Entroido do Ulla. La heterogeneidad de los dibujos que acompañan a las narrativas del alumnado dificulta su clasificación, pero casi un $80 \%$ de los dibujos tienen algún tipo de relación con algún elemento representativo.

El alumnado atribuye gran importancia para el municipio a que se desarrollen relaciones vecinales comunitarias (el 83\% lo considera una cuestión muy importante), seguido del atractivo turístico (el 52\% opina que la visita de gente de fuera de ayuntamiento es poco o nada importante). Casi tres cuartas partes con- sidera muy importante la transmisión de tradiciones y costumbres en la gente nueva. Existen ciertas diferencias por segmentos etarios y por participación. Así, el grupo de 12 a 14 años (el que más participó) considera, unánimemente, muy importante que el vecindario se reúna y relacione (el porcentaje es algo menor entre los mayores de 14 años y mucho menor entre los menores de 12). Sucede algo similar con el nivel de importancia adjudicado al mantenimiento de las costumbres tradicionales, mayor a partir de los 12 años que en el de 9 a 11. El grupo experimental es el que más importancia otorga al aspecto comunitario y a la continuidad de la tradición por los jóvenes (Gráfico 7).

Los adultos coinciden en que la fiesta de los Xenerais permite mantener viva parte de las tradiciones del pueblo y transmitirlas a las nuevas generaciones (88\%). Opinión compartida tanto por mayores como por escolares. También consideran que esta tradición favorece que las relaciones entre los habitantes de Vedra $(82 \%)$ y, tal y como se registró en el grupo de discusión, constituye una oportunidad para incrementar la visibilidad de Vedra como atractivo turístico, lo que podría contribuir a la continuidad de la tradición. Del grupo de discusión también se desprende que conseguir que perviva el entroido, con todos sus elementos y singularidades, pasa por difundirlo y visibilizarlo: "lo que no se ve no existe". Consideran, también, que son los niños/ as los/as encargados/as de seguir reproduciendo esta tradición, 


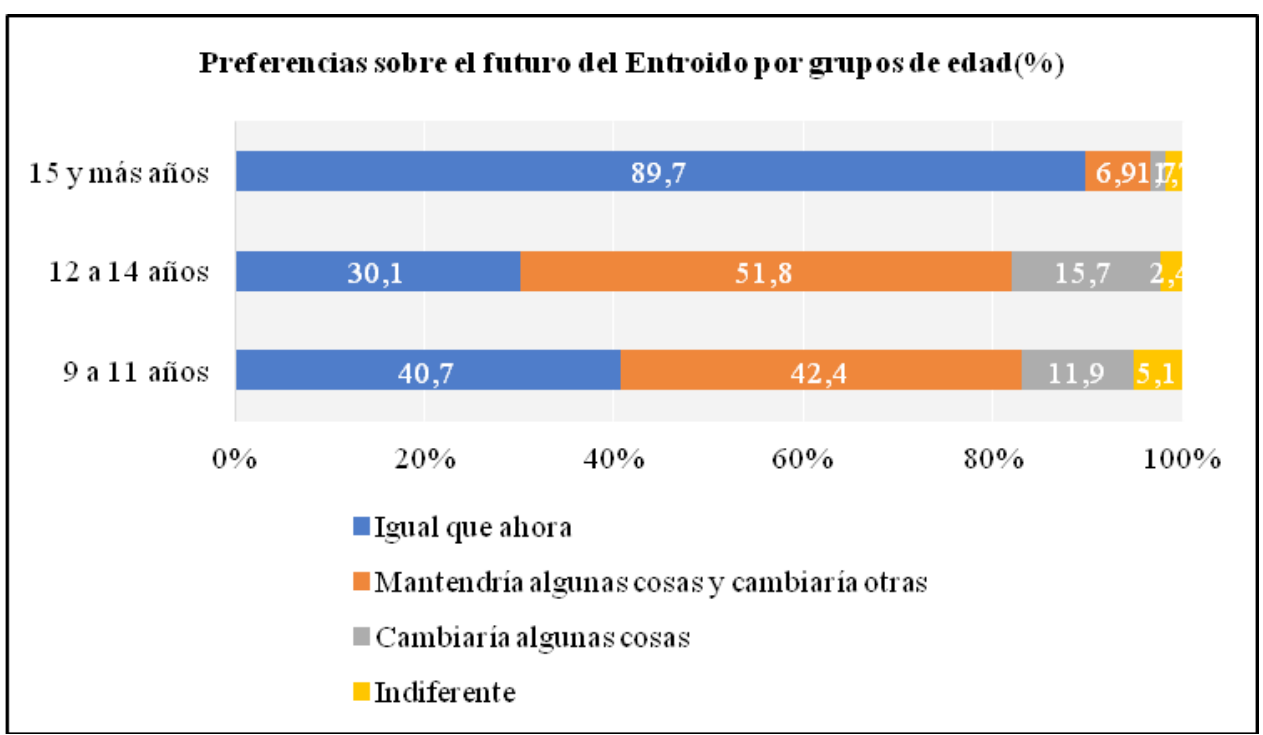

Gráfico 6. Preferencias del alumnado sobre el futuro del Entroido por grupos de edad

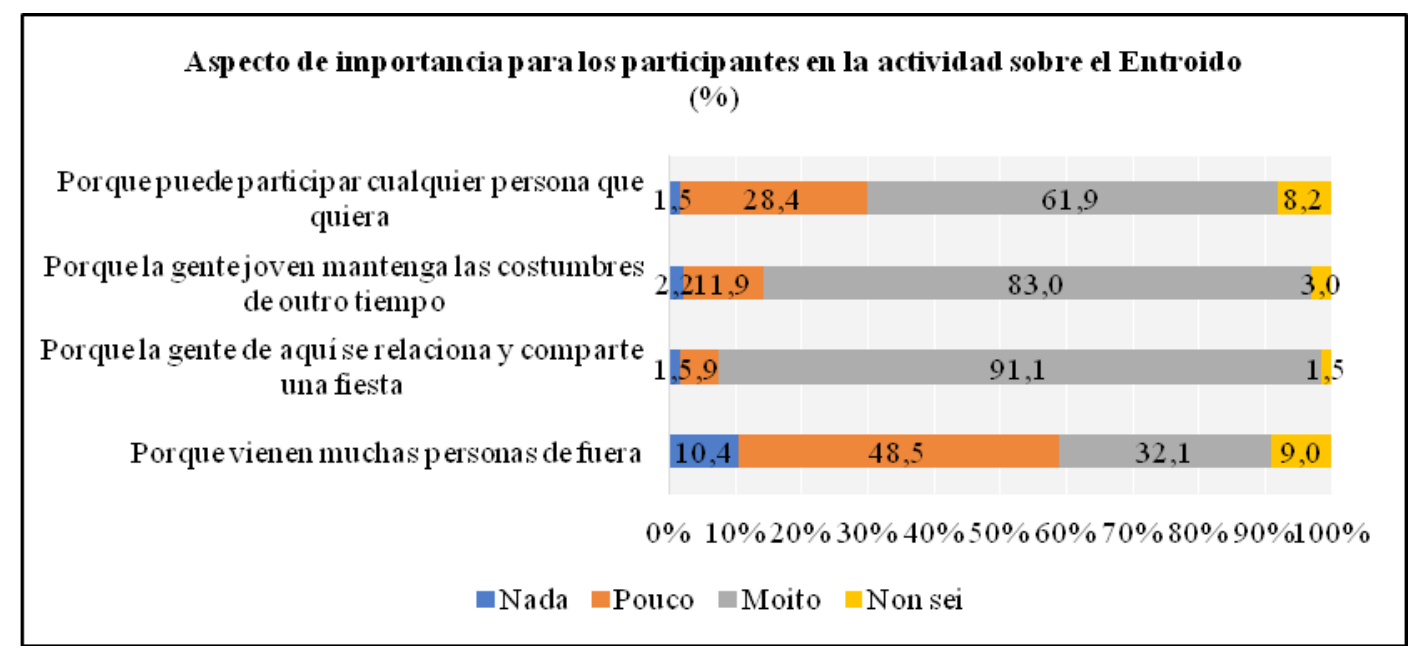

Gráfico 7. Aspectos de importancia para el grupo experimental sobre el Entroido

por lo que hacerlos partícipes de ella, enseñarles sus características y promover su interés y gusto por ella es otra de las líneas a seguir. En este sentido, resulta de importancia establecer un nexo de unión y trabajo entre el colegio y la comunidad, a fin de optimizar recursos y facilitar que toda la población pueda disfrutar de ellos y mantenerlos vivos. En este sentido, el grupo de discusión deja patente que las personas mayores pueden aportar su experiencia y anécdotas para enriquecer los aprendizajes escolares y contribuir a mantener vivas las tradiciones.

Objetivo 3. Caracterizar el significado en la construcción de una identidad compartida

Participación en el Entroido do Ulla de personas foráneas o nacidas en otro lugar

Existen ligeras diferencias en función de su participación en las actividades, pero bastante coincidencia entre los diferentes grupos de edad. Casi dos tercios del alumnado considera que el Entroido da Ulla es una festividad abierta a la participación de cualquiera, sin importar su origen (62\%). Pero con un elevado porcentaje de indiferentes (33\%). El grupo experimental se muestra ligeramente más abierto $(62,8 \%)$ que el de control $(55,6 \%)$.
Los adultos aprueban la participación de personas foráneas en mayor proporción que los menores (75\%). Coinciden en que es una fiesta abierta a quienes quieran participar. Como se manifestó en el grupo de discusión, incrementar la participación potencia su difusión y ayuda a conservar y mantener la tradición. Pero también, participar de esta fiesta es una forma de implicarse más en el pueblo e integrarse entre su vecindario, ya que permite establecer lazos y formas de colaboración.

\section{Participación de la mujer como protagonista}

La incorporación de la mujer como protagonista en esta fiesta tradicional se inició hace tres décadas. El grupo experimental de los escolares conoce esta incorporación reciente $(85,3 \%)$ en mayor medida que el grupo de control $(65,6 \%)$. La mayoría aprueban que las mujeres participen como Xeneralas (82\%), resultando prácticamente residual (5\%) el porcentaje que no está de acuerdo. Tanto varones como mujeres están claramente a favor de las Xeneralas en el Entroido, aunque los hombres cuentan con un porcentaje mayor de indiferencia: $16,9 \%$ frente al $4,7 \%$ de las mujeres $\left[\chi^{2}=8,817(\right.$ Sig. $=.012)$ para el cruce entre p15 y género]. 
Casi tres cuartas partes de los escolares encuestados consideran que la participación de las mujeres como Xeneralas fue fruto de la movilización de las propias mujeres, mientras que una cuarta parte se divide entre quienes piensan que esto se ha debido a un consenso general (15\%) y un $11 \%$ que lo atribuye a la ley de igualdad. Las principales diferencias se producen nuevamente en función del género y a la participación o no en las actividades (y, por lo tanto, en los grupos de edad) $\left[\chi^{2}=9,118(\right.$ Sig. $=.010)$ para el cruce entre p16 y género; $\chi^{2}=11,376($ Sig. $=.023)$ para el cruce entre p16 y edad; $\chi^{2}=11,040$ (Sig.= .026) para el cruce entre p16 y p1]. El grupo de control otorga más peso a algún tipo de ley de igualdad, una opción muy reducida en el experimental. El grupo de entre 12 y 14 años atribuye menor importancia a esta explicación y, la totalidad, considera que ha sido fruto de una reivindicación conseguida por las mujeres. Por género, si bien ambos sectores consideran mayoritariamente que se deben a una reivindicación exitosa de las mujeres, entre los hombres hay respuestas más distribuidas, y mayor importancia a algún tipo de acuerdo social para el cambio de la tradición.

Mayores y escolares coinciden, mayoritariamente, que la participación de mujeres como Xeneralas es porque algunas de ellas reclamaron su derecho a poder hacerlo. Existe un consenso generalizado en que las mujeres pueden, y deben, participar de la fiesta con los mismos derechos que los hombres. La totalidad de los adultos/as encuestados están de acuerdo con la participación de las mujeres como Xeneralas, algo que se ha corroborado también en el grupo de discusión.

\section{Conclusión}

Se ha pretendido responder a la pregunta de investigación: ¿en qué medida es posible realizar un proyecto de centro sobre educación patrimonial que implique a la comunidad local, a pesar de las limitaciones derivadas de las rutinas escolares? Los resultados parecen demostrar que el proyecto, a pesar de no encajar en el ritmo lectivo y la limitada implicación del profesorado, consiguió superar los obstáculos y crear una comunidad solidaria en la construcción de una identidad territorial, a partir del patrimonio local. Ha sido posible porque hay una dinámica anterior, que facilita poder conseguir la participación voluntaria de jóvenes y adultos fuera del horario escolar. Su fortaleza reside en la capacidad de continuar con alumnado diferente en sucesivos cursos escolares; crear diálogo intergeneracional, destacando que las memorias son fundamentales porque contribuyen a dar significado al territorio; convertir a los escolares en agentes patrimonializadores, no expertos, pero fundamentales en la construcción social del patrimonio y la decisión activa sobre el mismo (Cobas, 2016), con responsabilidad de conservar y transmitir una tradición viva en su territorio; así como concienciar a la comunidad sobre el papel del patrimonio local como instrumento de cohesión social (San Pedro y López, 2017). Repensar el patrimonio desde la mirada educativa implica entender la educación como proyecto de futuro que, bajo esta perspectiva, pueda contribuir al (re)descubrimiento del patrimonio local, muchas veces ignorado o despreciado, a través de un aprendizaje vivencial (estrechando lazos entre escuela y comunidad) y contextualizado (manejando distintos tipos de fuentes, fomentando un pensamiento histórico e identificando el valor histórico de ciertos elementos).

\section{Referencias}

Barraza, L. y Castaño, C. (2012). ¿Puede la enseñanza de la ciencia ayudar a construir una sociedad sostenible? Profesorado. Revista de Currículum y Formación del Profesorado, 16(2), 45-58.
Bisquerra Alzina, R. (1989). Métodos de investigación educativa. Guía práctica. Barcelona: CEAC.

Buendía, L.; Colás, P. y Hernández, F. (1998). Métodos de investigación en psicopedagogía. Madrid: McGraw-Hill.

Carrión Gútiez, A. (coord.) (2015). Plan Nacional de Salvaguarda del Patrimonio Cultural Inmaterial. Madrid: Ministerio de Educación, Cultura y Deporte.

Cobas Fernández, I. (2016). El concepto de paisaje cultural como recurso para la educación patrimonial en la educación secundaria obligatoria. CAPA. Cadernos de Arqueoloxía e Patrimonio, 37. Santiago de Compostela: CSIC-Instituto de Ciencias del Patrimonio.

Cuenca-López, J. M. y Estepa-Giménez, J. (2017). Educación patrimonial para la inteligencia territorial y emocional de la ciudadanía. MIDAS [Online], 8, [http://journals.openedition. org/midas/1173].

Deslauriers, J-P. (2004). Investigación cualitativa. Guía práctica. Pereira: Papiro.

Domínguez Almansa, A. y López Facal, R. (2017). Formación de maestros y educación patrimonial. Estudios Pedagógicos, 43(4), 49-68.

Elfer, Ch. (2011). Place-Based Education: Making the case for an Investigation of Historical Precedents. Curriculum History, 1, 1-15

Fontal Merillas, O. (2003). La educación patrimonial: teoría y práctica para el aula, el museo e internet. Gijón: Trea.

Fontal Merillas, O. y Gómez-Redondo, C. (2015). Evaluación de programas educativos que abordan los procesos de patrimonialización, Didáctica de las Ciencias Experimentales y Sociales, $29,89-118$.

Gómez Redondo, C. (2012). Identización: La construcción discursiva del individuo. Arte, Individuo y Sociedad, 24(1), 21-37.

Hatton-Yeo, A. y Ohsako, T. (eds.). (2001). Programas Intergeneracionales: política pública e implicaciones de la investigación. Una perspectiva internacional. Hamburgo: Instituto de la UNESCO para la Educación.

López-Facal, R. (2019). Identidade, identificación e relato sobre Galicia como nación, eHumanista/IVITRA, 15, 225-235.

Madariaga-Orbea, J. Mª, Gillate, I., Ibañez-Etxeberria, A. y Molero, B. (2018) Heritage education in informal contexts. Motivation and self-concept. Cultura y Educación, 30(3), 584-599.

Marqués Valea, X. (2013). Tendendo pontes, atando cabos. Revista galega de educación, 60, 66-67.

Marqués Valea, X. (2014). O patrimonio onomástico persoal. Algunhas reflexións sobre o seu valor educativo e uso didáctico. Revista galega de educación, 65. [http:/ / www.edu.xunta. gal/eduga/373/foro/o-patrimonio-onomastico-persoal].

Martín Cáceres, M. y Cuenca López, J. M. (2015). Educomunicación del patrimonio, Educatio Siglo XXI, 33(1), 33-54.

Mcmillan, J. y Schumacher, S. (2005). Investigación educativa. Una introducción conceptual. Madrid: Pearson Educación.

Peterson, R. B. (2018). Taking it to the city: urban-placed pedagogies in Detroit and Roxbury. Journal of Environmental Studies and Sciences, 8(3), 326-342.

San Pedro Veledo, B. y López Manrique, I. (2017). El profesorado de ciencias sociales en contextos rurales de Asturias: concepciones y uso de recursos educativos. Aula Abierta, 45, 33-40.

Sánchez Contreras M. F. y Murga-Menoyo, M. A. (2019). Place-Based Education: Una estrategia para la sostenibilización curricular de la educación superior. Bordón. Revista de Pedagogía. 71(2), 154-174.

Sobel, D. (2004). Place-based education: Connecting classroom and community. Nature and Listening, 4, 1-7.

UNESCO (2003). ¿Qué es el patrimonio cultural inmaterial?. [http:/ / www.culturaydeporte.gob.es/dam/jcr:49a3eaa1-d333-48aa- 
a02b-e6e07c2549aa/que-es-patrimonio-cultural-inmaterial. pdf].

UNESCO (2012) Exploring Sustainable Development: A Multiple-Perspective Approach. Education for Sustainable Development. Action Learning \& Training Tools, 3. Paris: UNESCO.
Woodhouse, J. L. y Knapp, C. E. (2000). Place-Based Curriculum and Instruction: Outdoor and Environmental Education Approaches. ERIC Digest [https://www.ericdigests.org/20013/place.htm]. 